\title{
Efficient Algorithms for Approximating Polygonal Chains*
}

\author{
P. K. Agarwal $^{1}$ and K. R. Varadarajan ${ }^{2}$ \\ ${ }^{1}$ Center for Geometric Computing, \\ Department of Computer Science, Duke University, \\ Durham, NC 27708-0129, USA \\ pankaj@cs.duke.edu \\ 2 DIMACS, Rutgers University, \\ Piscataway, NJ 08854, USA \\ krv@dimacs.rutgers.edu
}

\begin{abstract}
We consider the problem of approximating a polygonal chain $C$ by another polygonal chain $C^{\prime}$ whose vertices are constrained to be a subset of the set of vertices of $C$. The goal is to minimize the number of vertices needed in the approximation $C^{\prime}$. Based on a framework introduced by Imai and Iri [25], we define an error criterion for measuring the quality of an approximation. We consider two problems. (1) Given a polygonal chain $C$ and a parameter $\varepsilon \geq 0$, compute an approximation of $C$, among all approximations whose error is at most $\varepsilon$, that has the smallest number of vertices. We present an $O\left(n^{4 / 3+\delta}\right)$-time algorithm to solve this problem, for any $\delta>0$; the constant of proportionality in the running time depends on $\delta$. (2) Given a polygonal chain $C$ and an integer $k$, compute an approximation of $C$ with at most $k$ vertices whose error is the smallest among all approximations with at most $k$ vertices. We present a simple randomized algorithm, with expected running time $O\left(n^{4 / 3+\delta}\right)$, to solve this problem.
\end{abstract}

\section{Introduction}

Let $C=\left\langle p_{1}, \ldots, p_{n}\right\rangle$ denote a polygonal chain whose vertices are $p_{1}, \ldots, p_{n}$ and whose edges are $p_{1} p_{2}, p_{2} p_{3}, \ldots, p_{n-1} p_{n}$. A polygonal chain $C^{\prime}=\left\langle p_{i_{1}}=p_{1}, \ldots, p_{i_{k}}=p_{n}\right\rangle$ is an approximation of $C$ if $i_{1}<\cdots<i_{k}$ (Fig. 1).

* Work on this paper has been supported by an NSF Grant CCR-93-01259, an Army Research Office MURI Grant DAAH04-96-1-0013, an NYI award, and matching funds from the Xerox Corporation. 


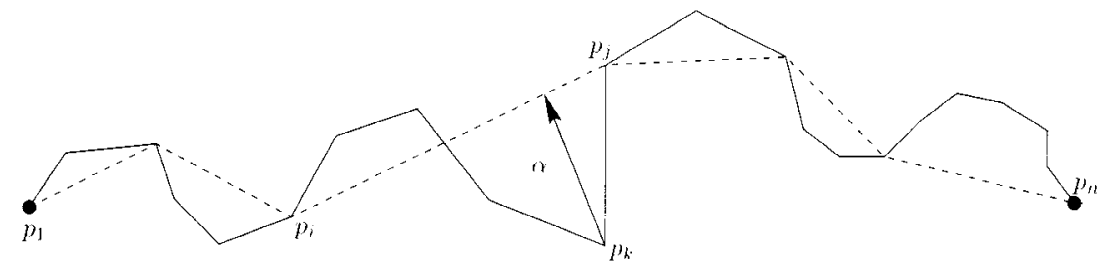

Fig. 1. A polygonal chain (in bold) and its approximation (dashed); the error, under the Euclidean metric, of segment $p_{i} p_{j}$ is $\alpha$.

Let $d(\cdot, \cdot)$ be a distance function defined on points in the plane. We define the error of a line segment $p_{i} p_{j}$ connecting two points of $C$, where $i<j$, to be

$$
\Delta\left(p_{i} p_{j}\right)=\max _{i \leq k \leq j} d\left(p_{k}, p_{i} p_{j}\right)
$$

where the distance between a point $p$ and a segment $e$ is $d(p, e)=\min _{q \in e} d(p, q)$. The error of an approximation $C^{\prime}$ of $C$ is

$$
\Delta_{C}\left(C^{\prime}\right)=\max _{1 \leq j<k} \Delta\left(p_{i_{j}} p_{i_{j+1}}\right)
$$

the maximum of the errors of each of the edges of $C^{\prime}$. We will omit the subscript for $\Delta$ when the chain $C$ that is being approximated is clear from the context. We call $C^{\prime}$ an $\varepsilon$-approximation of $C$ if $\Delta_{C}\left(C^{\prime}\right) \leq \varepsilon$. We consider two problems:

1. Min-\# problem: Given a polygonal chain $C$ and a real number $\varepsilon \geq 0$, compute an $\varepsilon$-approximation of $C$ that uses the the smallest number of vertices among all $\varepsilon$-approximations of $C$.

2. Min- $\varepsilon$ problem: Given a polygonal chain $C$ and an integer $k$, compute an approximation of $C$ with at most $k$ vertices that minimizes the error over all approximations of $C$ that have at most $k$ vertices.

\subsection{Motivation and Previous Results}

Polygonal curves are often used to represent boundaries of planar objects in cartography, computer graphics, pattern recognition, etc. The general problem of approximating a polygonal curve by a coarser one is of fundamental importance, and has been studied in disciplines such as geographic information systems [7], [11], [15], [21], [29], [31], digital image analysis [5], [24], [28], and computational geometry [8], [16], [18], [19], [25], [33], [35]. The problems considered fall in one of two categories, depending on whether the vertices of the approximating chain are required to be a subset of the vertices of the input chain. Within each category, min-\# and min- $\varepsilon$ problems have been studied under different error criteria.

We first mention some of the algorithms that compute approximations whose vertices are not restricted to being on the input chain. A well-studied problem in this category is to compute a piecewise-linear function approximating an $n$-point planar data set, using 
the uniform metric to measure the error. This is in fact a special case of a whole class of problems in approximation theory that seek to fit a set of data using a spline function under some metric. (See texts discussing approximation theory such as [6], [9], [12], and [14].) Hakimi and Schmeichel [20] give an $O(n)$-time algorithm for the min-\# version of the problem and an $O\left(n^{2} \log n\right)$-time algorithm for the min- $\varepsilon$ version. Goodrich [18] gives an improved $O(n \log n)$-time algorithm for the min- $\varepsilon$ version. The min-\# problems in this category closely resemble the problem of computing a minimum link path in a polygonal domain, which has been studied extensively; see [3], [19], [22], and [25] for a sample. Hershberger and Snoeyink [22] give an efficient algorithm for computing a minimum link path of a given homotopy. In this problem we are given two points $s$ and $t$ in a polygonal region $R$, which may have holes, and a representative path $\pi$ between $s$ and $t$ in $R$, and we want to compute a minimum-link path in $R$ between $s$ and $t$ that is homotopic to $\pi$. Homotopy considerations are important when polygonal approximation occurs in the context of other features; see the paper by de Berg et al. [13]. Sometimes, we also want the approximation of a given polygonal chain to be simple, that is, nonself-intersecting. This requirement seems to make the problems intractable. Guibas et al. [19] show that the problem of computing a minimum-link simple approximation of a simple polygon is NP-hard (for a particular error criterion). They also show that a version of the problem of approximating a polygonal subdivision (as opposed to just a single polygonal chain) is NP-hard.

We now turn to the methods for computing approximations whose vertices are restricted to being a subset of the vertices of the input chain. This is the class to which this paper belongs. The restricted case is important because in many applications, attributes such as color are associated with the vertices of the input chain. Moreover, in the unrestricted case, we have to deal with the issue of the precision needed to represent vertices in the approximation (see [26]). One of the oldest and most popular algorithms in cartography is the Douglas-Peucker algorithm [15], a heuristical approach that computes an approximation within a prespecified error. Hershberger and Snoeyink [23] showed that the Douglas-Peucker algorithm can be implemented in $O\left(n \log ^{*} n\right)$ time, where $\log ^{*}$ is the iterated logarithm function [10]. Imai and Iri [25] present a unified approach to polygonal approximation problems by formulating them in terms of graph theory, and present algorithms for approximating simple polygonal chains under a number of error criteria. Algorithms with the same flavor are also described by Chan and Chin [8], Melkman and O'Rourke [33], and Toussaint [36]. Most of these algorithms run in $\Omega\left(n^{2}\right)$ time. It appears as though the problems in this category become intractable if we require the approximation to be simple, or to be homotopic to the original chain; see [13] for a discussion.

\subsection{Our Results}

In this paper we study the min-\# and min- $\varepsilon$ problems under the $L_{1}$ and uniform (also known as Chebyshev) metric. Recall that the distance between two points $p=\left(p_{x}, p_{y}\right)$ and $q=\left(q_{x}, q_{y}\right)$ in the $L_{1}$ metric is

$$
d(p, q)=\left|p_{x}-q_{x}\right|+\left|p_{y}-q_{y}\right|
$$


and the distance under the uniform metric is

$$
d(p, q)= \begin{cases}\left|p_{y}-q_{y}\right| & \text { if } p_{x}=q_{x} \\ \infty & \text { otherwise. }\end{cases}
$$

Note that the distance between a point $p$ and a segment $e$ under the uniform metric is the vertical distance between $p$ and $e$ if the $x$-projections of $p$ and $e$ intersect, and infinity otherwise. The two main results are the following:

- An $O\left(n^{4 / 3+\delta}\right)$-time deterministic algorithm, for any $\delta>0$, for the min-\# problem under the $L_{1}$ metric.

- An $O\left(n^{4 / 3+\delta}\right)$ expected-time randomized algorithm, for any $\delta>0$, for the min- $\varepsilon$ problem under the $L_{1}$ metric.

Our techniques also generalize to certain other metrics that approximate the Euclidean metric closely. Section 2 discusses preliminaries needed by our algorithms and the main idea used. In order to present the techniques clearly, we describe in Section 3 the min-\# algorithm for an $x$-monotone chain under the uniform metric. In Section 4 we show how the techniques used in Section 3 can be generalized to solve the min-\# problem for any polygonal chain under the $L_{1}$ metric. In Section 5 we describe a simple randomized algorithm for the min- $\varepsilon$ problem under the $L_{1}$ metric. We offer our conclusions in Section 6.

\section{Preliminaries}

Imai and Iri's Algorithm. Since our algorithm is based on Imai and Iri's general framework for polygonal chain approximation, we first describe their approach. Let $C$ be the polygonal chain $\left\langle p_{1}, \ldots, p_{n}\right\rangle$, and let $\varepsilon>0$ be a given error bound. Assume that we have fixed a distance function on points in the plane. We define an unweighted, directed graph $G_{\varepsilon}(C)=\left(V, E_{\varepsilon}\right)$, where $V=\left\{p_{1}, \ldots, p_{n}\right\}$ and

$$
E_{\varepsilon}=\left\{\left(p_{i}, p_{j}\right) \mid 1 \leq i<j \leq n \text {, and } \Delta\left(p_{i} p_{j}\right) \leq \varepsilon\right\} .
$$

Figure 2 illustrates this definition for an $x$-monotone chain under the uniform metric. We sometimes denote $G_{\varepsilon}(C)$ by $G_{\varepsilon}$ when the underlying chain $C$ is clear from the context.

An $\varepsilon$-approximation of $C$ corresponds to a path from $p_{1}$ to $p_{n}$ in $G_{\varepsilon}$, and an $\varepsilon$ approximation with the minimum number of vertices corresponds to a shortest path

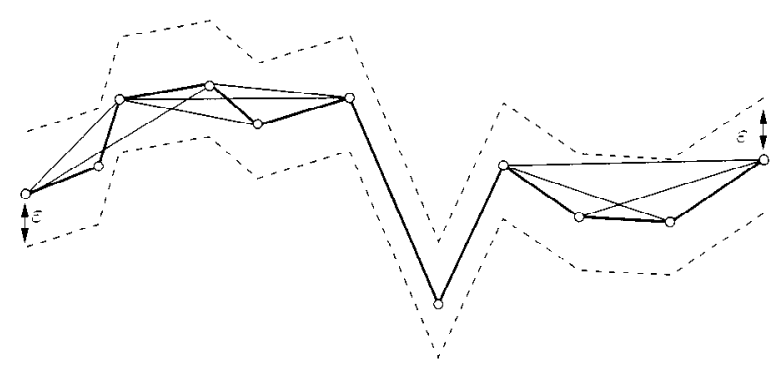

Fig. 2. The graph $G_{\varepsilon}$ for an $x$-monotone chain under the uniform metric; solid segments are graph edges. 
from $p_{1}$ to $p_{n}$ in $G_{\varepsilon}$. The shortest path, which is the solution to the min-\# problem, can be found by a breadth first search in $G_{\varepsilon}$ using $O\left(\left|E_{\varepsilon}\right|\right)=O\left(n^{2}\right)$ time. The min- $\varepsilon$ problem involves computing the smallest value of $\varepsilon$ for which there is a path in $G_{\varepsilon}$ from $p_{1}$ to $p_{n}$ consisting of at most $k$ vertices. Let $\varepsilon^{*}$ denote this smallest value. Let $\varepsilon_{1} \leq \varepsilon_{2} \cdots$ be the values of $\Delta\left(p_{i} p_{j}\right)$ for $1 \leq i<j \leq n$. Note that $G_{\varepsilon}$ remains the same for all $\varepsilon \in\left[\varepsilon_{i}, \varepsilon_{i+1}\right)$. Therefore, the min- $\varepsilon$ problem reduces to finding the smallest $\varepsilon_{i}$ for which $G_{\varepsilon_{i}}$ contains a path from $p_{1}$ to $p_{n}$ with at most $k$ vertices. Since $E_{\varepsilon_{i}} \subseteq E_{\varepsilon_{i+1}}, \varepsilon^{*}$ can be computed by a binary search on the $\varepsilon_{i}$ 's, using the min-\# algorithm as the decision procedure.

Using Imai and Iri's approach naively, any algorithm for the min-\# problem takes $\Omega\left(n^{2}\right)$ time, as it needs to construct the graph $G_{\varepsilon}$, which can have $\Theta\left(n^{2}\right)$ edges. Chan and Chin [8], Imai and Iri [25], Melkman and O'Rourke [33], and Toussaint [36] give quadratic or near-quadratic-time algorithms for constructing $G_{\varepsilon}$ under various error criteria. The running time for the min- $\varepsilon$ problems is governed by the time it takes to compute the errors $\Delta\left(p_{i} p_{j}\right)$ of the segments, and the time for $O(\log n)$ applications of the min-\# decision procedure. Hence, these algorithms also take $\Omega\left(n^{2}\right)$ time.

Compact Representation of $G_{\varepsilon}$. If we are aiming for a subquadratic algorithm, we cannot compute the graph $G_{\varepsilon}$ explicitly. Instead, we construct a compact representation of $G_{\varepsilon}$. Let $G=(V, E)$ be a directed graph, and let

$$
\mathcal{G}=\left\{G_{1}=\left(V_{1}, E_{1}\right), \ldots, G_{l}=\left(V_{l}, E_{l}\right)\right\}
$$

be a family of subgraphs of $G$. We say that $\mathcal{G}$ is a clique cover of $G$ if the following conditions hold:

1. Each $G_{i}$ is a complete bipartite graph. If $V_{i_{1}}, V_{i_{2}} \subseteq V_{i}$ are the two vertex classes of $G_{i}$, then every edge of $G_{i}$ must be directed from a vertex in $V_{i_{1}}$ to a vertex in $V_{i_{2}}$.

2. $E=E_{1} \cup \cdots \cup E_{l}$.

3. $E_{i} \cap E_{j}=\emptyset$ for $i \neq j$.

Since each $G_{i}$ is a bipartite clique, we can represent it compactly by specifying its vertex classes $V_{i_{1}}$ and $V_{i_{2}}$; this takes $O\left(\left|V_{i}\right|\right)$ space. The edges are now defined implicitly. We define the size of the clique cover $\mathcal{G}$, denoted by $|\mathcal{G}|$, to be $\sum_{i=1}^{l}\left|V_{i}\right|$; this is the space we require to represent $G$ compactly. The notion of "compressing" graphs using clique covers is pursued by Feder and Motwani [17], who use clique covers of graphs to speed up a number of graph algorithms.

Proposition 2.1. Let $\mathcal{G}=\left\{G_{1}, \ldots, G_{l}\right\}$ be a clique cover of a directed graph $G$. Given two vertices $u$ and $v$ in $G$, we can compute a shortest path between $u$ and $v$ in $G$ in $O(|\mathcal{G}|+|V|)$ time.

Proof. We define a new graph $H$ whose vertex set is $V \cup\left\{g_{1}, \ldots, g_{l}\right\}$, where $g_{1}, \ldots, g_{l}$ are additional vertices. If $(u, v)$ is an edge in $G$, and if $(u, v)$ belongs to $G_{i}$, then we add the edges $\left(u, g_{i}\right)$ and $\left(g_{i}, v\right)$ in $H$. There are $O(|\mathcal{G}|+|V|)$ vertices and $O(|\mathcal{G}|)$ edges in $H$. We compute a shortest path between $u$ and $v$ in $H$. If the procedure returns the path $\left\langle u_{1}=u, g_{1}, u_{2}, g_{2}, \ldots, g_{k}, u_{k+1}=v\right\rangle$ in $H$, then we return $\left\langle u_{1}, u_{2}, \ldots, u_{k+1}\right\rangle$ as the 
shortest path between $u$ and $v$ in $G$. The correctness of the procedure is straightforward. This computation takes time proportional to $|\mathcal{G}|+|V|$.

\section{The Min-\# Algorithm under the Uniform Metric}

Let $C=\left\langle p_{1}, \ldots, p_{n}\right\rangle$ be an $x$-monotone polygonal chain, and let $\varepsilon$ be a given error bound. In this section we present an algorithm that solves the min-\# problem for $C$ under the uniform metric. Our algorithm computes a shortest path between $p_{1}$ and $p_{n}$ in the graph $G_{\varepsilon}$ defined above. In order to do this efficiently, we first compute a clique cover of $G_{\varepsilon}$, and then use Proposition 2.1 to compute a shortest path from $p_{1}$ to $p_{n}$ in $G_{\varepsilon}$.

In the remainder of this section we describe an $O\left(n^{4 / 3+\delta}\right)$-time divide-and-conquer algorithm for constructing a clique cover $\mathcal{G}$ of $G_{\varepsilon}=G_{\varepsilon}(C)$. Let $C_{1}$ be the chain $\left\langle p_{1}, \ldots, p_{\lfloor n / 2\rfloor}\right\rangle$ and let $C_{2}$ be the chain $\left\langle p_{\lfloor n / 2\rfloor+1}, \ldots, p_{n}\right\rangle$. Recall that $\Delta\left(p_{i} p_{j}\right)=$ $\max _{i \leq k \leq j} d\left(p_{k}, p_{i} p_{j}\right)$, and that a pair $\left(p_{i}, p_{j}\right) \in E_{\varepsilon}$ if $\Delta\left(p_{i} p_{j}\right) \leq \varepsilon$. Hence, if $\left(p_{i}, p_{j}\right) \in$ $E_{\varepsilon}$ and $p_{i}, p_{j} \in C_{1}$ (resp. $\left.p_{i}, p_{j} \in C_{2}\right)$, then $\left(p_{i}, p_{j}\right)$ is an edge in $G_{\varepsilon}\left(C_{1}\right)\left(\right.$ resp. $\left.G_{\varepsilon}\left(C_{2}\right)\right)$. We recursively compute clique covers $\mathcal{G}_{1}, \mathcal{G}_{2}$ of $G_{\varepsilon}\left(C_{1}\right)$ and $G_{\varepsilon}\left(C_{2}\right)$, respectively. In the merge step we compute a clique cover $\mathcal{G}_{12}$ of the edges

$$
E_{12}=\left\{\left(p_{i}, p_{j}\right) \in G_{\varepsilon}(C) \mid p_{i} \in C_{1}, p_{j} \in C_{2}\right\} .
$$

That is, $\mathcal{G}_{12}$ is a clique cover of the edges of $G_{\varepsilon}(C)$, one of whose endpoints is in $C_{1}$ and the other in $C_{2} . \mathcal{G}_{1} \cup \mathcal{G}_{2} \cup \mathcal{G}_{12}$ is a clique cover of $G_{\varepsilon}(C)$. Before describing how to compute $\mathcal{G}_{12}$, we need a few definitions and preliminary lemmas.

If $\left(x_{i}, y_{i}\right)$ denotes the coordinates of $p_{i}$, let $p_{i}^{-}$be the point $\left(x_{i}, y_{i}-\varepsilon\right)$, and let $p_{i}^{+}$be the point $\left(x_{i}, y_{i}+\varepsilon\right)$. It is easy to see that $\left(p_{i}, p_{j}\right)$ is an edge of $G_{\varepsilon}(C)$ if and only if for every $k, i \leq k \leq j$, the vertical segment $p_{k}^{-} p_{k}^{+}$intersects the segment $p_{i} p_{j}$. For $p_{i} \in C_{1}$, we define cone $\left(p_{i}\right)$ to be the following subset of rightward directed rays emanating from $p_{i}$ : a ray $\rho$ belongs to cone $\left(p_{i}\right)$ if $\rho$ intersects $p_{k}^{-} p_{k}^{+}$, for every $i \leq k \leq\lfloor n / 2\rfloor$. (Figure 3 illustrates the definition of cone $\left(p_{i}\right)$.) We regard cone $\left(p_{i}\right)$ not only as a set of rays, but as a set of points as well. Note that the interior of $\operatorname{cone}\left(p_{i}\right)$ does not contain any $p_{k}^{-}, p_{k}^{+}$, for any $i \leq k \leq\lfloor n / 2\rfloor$, and for any ray $\rho \in \operatorname{cone}\left(p_{i}\right), d\left(p_{k}, \rho\right) \leq \varepsilon$, for $i \leq k \leq\lfloor n / 2\rfloor$. Symmetrically, for a vertex $p_{j}$ of $C_{2}$, we define cone $\left(p_{j}\right)$ to be the following collection of leftward directed rays emanating from $p_{j}$ : a ray $\rho$ belongs to $\operatorname{cone}\left(p_{j}\right)$ if it intersects $p_{k}^{-} p_{k}^{+}$, for $\lfloor n / 2\rfloor+1 \leq k \leq j$.

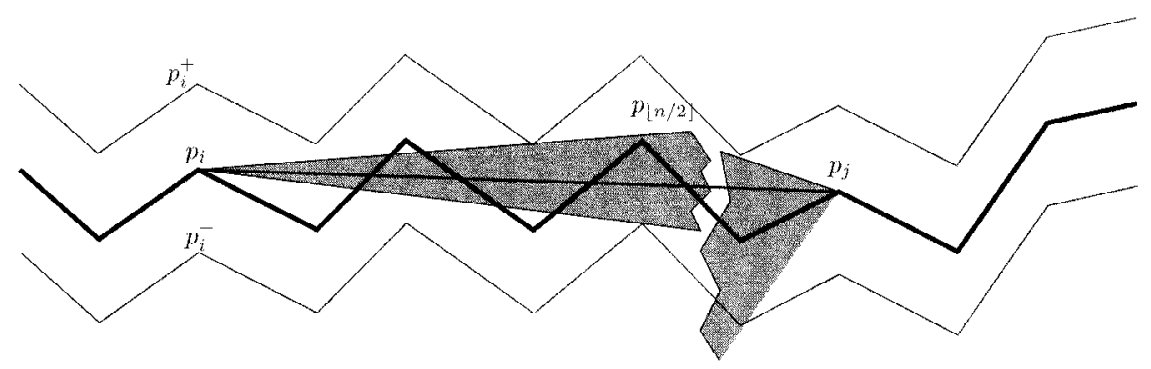

Fig. 3. cone $\left(p_{i}\right)$ and cone $\left(p_{j}\right)$; the edge $\left(p_{i}, p_{j}\right)$ is in $G_{\varepsilon}$ because $p_{j} \in \operatorname{cone}\left(p_{i}\right)$ and $p_{i} \in \operatorname{cone}\left(p_{j}\right)$ 
The boundary of a convex polygon $Q$ can be divided into two $x$-monotone convex chains at its leftmost and rightmost vertices. The chain lying above (resp. below) the line through the $x$-extremal vertices is called the upper (resp. lower) boundary of $Q$. Let $L_{i}$ denote the lower boundary of the convex hull of $\left\{p_{i}^{+}, \ldots, p_{\lfloor n / 2\rfloor}^{+}\right\}$and $U_{i}$ the upper boundary of the convex hull of $\left\{p_{i}^{-}, \ldots, p_{\lfloor n / 2\rfloor}^{-}\right\}$. By definition of $\operatorname{cone}\left(p_{i}\right)$, its top ray is tangent to $L_{i}$ and its lower ray is tangent to $U_{i}$. Note that cone $\left(p_{i}\right)$ is empty if the tangent to $L_{i}$ is below the tangent to $U_{i}$.

Lemma 3.1. We can compute cone $\left(p_{k}\right)$, for every $1 \leq k \leq n$, in $O(n \log n)$ time.

Proof. We only describe the computation of cone $\left(p_{k}\right)$ for $1 \leq k \leq\lfloor n / 2\rfloor$; we can compute cone $\left(p_{k}\right)$ for $\lfloor n / 2\rfloor+1 \leq k \leq n$ symmetrically. Suppose that we have inductively computed cone $\left(p_{k}\right)$ for $i \leq k \leq n / 2$. Suppose also that we have computed $L_{i}$ and $U_{i}$. We first compute $L_{i-1}$ and $U_{i-1}$ from $L_{i}$ and $U_{i}$, respectively, in $O(\log n)$ time using standard techniques [32], [34]. We can then compute in $O(\log n)$ time the tangents to $L_{i-1}$ and $U_{i-1}$ from $p_{i-1}$, and thus cone $\left(p_{i-1}\right)$.

Using a standard duality transformation (that maps a point $(a, b)$ to the line $y=a x+b$, and a line $y=m x+c$ to the point $(-m, c))$, we can map the line supporting any ray $\rho$ to a point $\rho^{*}$. We refer to the point $\rho^{*}$ as the dual of the ray $\rho$. Let $\gamma_{i}$ denote the set of points dual to the rays in cone $\left(p_{i}\right) . \gamma_{i}$ is a line segment in the dual plane. Let

$$
\Gamma_{1}=\left\{\gamma_{i} \mid 1 \leq i \leq\lfloor n / 2\rfloor\right\}
$$

and

$$
\Gamma_{2}=\left\{\gamma_{i} \mid\lfloor n / 2\rfloor+1 \leq i \leq n\right\} .
$$

The following lemma is obvious.

Lemma 3.2. Let $p_{i}\left(\right.$ resp. $\left.p_{j}\right)$ be a vertex of $C_{1}$ (resp. $\left.C_{2}\right)$. Then $p_{j} \in \operatorname{cone}\left(p_{i}\right)$ and $p_{i} \in \operatorname{cone}\left(p_{j}\right)$ if and only if $\gamma_{i}$ and $\gamma_{j}$ intersect.

Lemma 3.3. Let $p_{i}\left(\right.$ resp. $\left.p_{j}\right)$ be a vertex of $C_{1}$ (resp. $\left.C_{2}\right)$. Then $\left(p_{i}, p_{j}\right) \in G_{\varepsilon}$ if and only if $\gamma_{i}$ and $\gamma_{j}$ intersect.

Proof. Suppose that $\gamma_{i}$ and $\gamma_{j}$ intersect. By Lemma 3.2, it follows that $p_{j} \in \operatorname{cone}\left(p_{i}\right)$ and $p_{i} \in \operatorname{cone}\left(p_{j}\right)$. Thus the ray $R\left(p_{i}, p_{j}\right)$ emanating from $p_{i}$ and passing through $p_{j}$ is in cone $\left(p_{i}\right)$. Therefore, this ray intersects the segment $p_{k}^{-} p_{k}^{+}$, for $i \leq k \leq\lfloor n / 2\rfloor$. Since the chain $C$ is $x$-monotone, the line segment $p_{i} p_{j}$ intersects the segment $p_{k}^{-} p_{k}^{+}$, for $i \leq k \leq\lfloor n / 2\rfloor$. By a similar argument, we can conclude that the line segment $p_{i} p_{j}$ also intersects the segments $p_{k}^{-} p_{k}^{+}$, for $\lfloor n / 2\rfloor+1 \leq k \leq j$. Thus, the line segment $p_{i} p_{j}$ intersects all the segments $p_{k}^{-} p_{k}^{+}$, for $i \leq k \leq j$, which means that $\left(p_{i}, p_{j}\right) \in G_{\varepsilon}$.

To prove the converse, assume that $\left(p_{i}, p_{j}\right) \in G_{\varepsilon}$. Then the line segment $p_{i} p_{j}$ intersects all the segments $p_{k}^{-} p_{k}^{+}$, for $i \leq k \leq j$. It follows that the ray $R\left(p_{i}, p_{j}\right)$ emanating from $p_{i}$ and passing through $p_{j}$ intersects all the segments $p_{k}^{-} p_{k}^{+}$, for $i \leq k \leq\lfloor n / 2\rfloor$. Thus the ray $R\left(p_{i}, p_{j}\right)$ is in cone $\left(p_{i}\right)$, and so $p_{j} \in \operatorname{cone}\left(p_{i}\right)$. By a similar argument, we conclude that $p_{i} \in$ cone $\left(p_{j}\right)$. Lemma 3.2 then implies that $\gamma_{i}$ and $\gamma_{j}$ intersect. 
By Lemma 3.3, the problem of computing $\mathcal{G}_{12}$ reduces to computing a family

$$
\mathcal{F}=\left\{\left(\Gamma_{11}, \Gamma_{21}\right), \ldots,\left(\Gamma_{1 u}, \Gamma_{2 u}\right)\right\}
$$

so that the following conditions hold:

1. $\Gamma_{1 i} \subseteq \Gamma_{1}, \Gamma_{2 i} \subseteq \Gamma_{2}$;

2. each segment in $\Gamma_{i 1}$ intersects every segment in $\Gamma_{i 2}$; and

3. for every pair of intersecting segments $\gamma_{1} \in \Gamma_{1}, \gamma_{2} \in \Gamma_{2}$ there is a unique $i$ such that $\gamma_{1} \in \Gamma_{1 i}$ and $\gamma_{2} \in \Gamma_{2 i}$.

We now describe a procedure to compute such a family $\mathcal{F}$. We construct the segmentintersection-searching data structure [2] on the set $\Gamma_{2}$, which can report the set of segments of $\Gamma_{2}$ intersecting a query segment in the plane, as a union of few pairwise disjoint subsets of $\Gamma_{2}$. This segment-intersection-searching data structure is a multilevel partition tree, each of whose nodes is associated with a so-called canonical subset of $\Gamma_{2}$. The total size of all canonical subsets in the tree is $O\left(n^{4 / 3+\delta}\right)$. For a query segment $e$, the query procedure selects $O\left(n^{1 / 3+\delta}\right)$ pairwise disjoint canonical subsets whose union consists of exactly those segments $\Gamma_{2}$ that intersect $e$. Using this structure, we can construct the family $\mathcal{F}$ as follows. We query the data structure with all segments of $\Gamma_{1}$. For each canonical subset $\Gamma_{2 i}$ of $\Gamma_{2}$, let $\Gamma_{1 i} \subseteq \Gamma_{1}$ be the set of segments whose output contained $\Gamma_{2 i}$. If $\Gamma_{1 i} \neq \emptyset$, we add the pair $\left(\Gamma_{1 i}, \Gamma_{2 i}\right)$ to the family $\mathcal{F}$.

The size of the resulting clique cover $\mathcal{G}_{12}$ of $E_{12}$ is $\sum_{i}\left(\left|\Gamma_{1 i}\right|+\left|\Gamma_{2 i}\right|\right)$, which is $O\left(n^{4 / 3+\delta}\right)$. The running time for computing $\mathcal{G}_{12}$ is dominated by the time to compute the family $\mathcal{F}$, which is $O\left(n^{4 / 3+\delta}\right)$.

Let $S(n)$ denote the size of the clique cover of $G_{\varepsilon}(C)$ computed by the entire algorithm. Then we have the recurrence

$$
S(n) \leq 2 S(n / 2)+c n^{4 / 3+\delta}
$$

for some constant $c$. This recurrence solves to $S(n)=O\left(n^{4 / 3+\delta}\right)$. An identical argument shows that the running time of the algorithm is $O\left(n^{4 / 3+\delta}\right)$.

Lemma 3.4. For any $\delta>0$, we can compute a clique cover of $G_{\varepsilon}(C)$ of size $O\left(n^{4 / 3+\delta}\right)$ in $O\left(n^{4 / 3+\delta}\right)$ time.

From Proposition 2.1, it follows that we can compute a shortest path between $p_{1}$ and $p_{n}$ in $G_{\varepsilon}$ in $O\left(n^{4 / 3+\delta}\right)$ time. Thus, we have the following result.

Theorem 3.5. For any $\delta>0$, we can solve the min-\# problem under the uniform metric for an $x$-monotone chain with $n$ vertices in $O\left(n^{4 / 3+\delta}\right)$ time.

\section{The Min-\# Algorithm under the $L_{1}$ Metric}

Let $C=\left\langle p_{1}, \ldots, p_{n}\right\rangle$ be a polygonal chain (which is not necessarily $x$-monotone), and let $\varepsilon$ be a given error bound. In this section we take the underlying metric to be the $L_{1}$ metric, and show how we can efficiently compute a clique cover of the graph $G_{\varepsilon}$ defined 


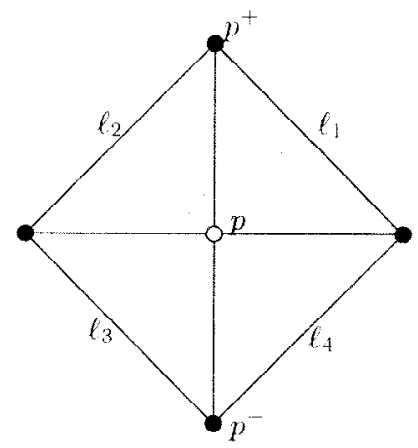

Fig. 4. The $L_{1}$ disk of radius $\varepsilon$ centered at $p$. $\Phi\left(\ell_{1}\right)=\Phi\left(\ell_{3}\right)=-\pi / 4$ and $\Phi\left(\ell_{2}\right)=\Phi\left(\ell_{4}\right)=\pi / 4$. Any line $\ell$, with $-\pi / 4 \leq \Phi(\ell) \leq \pi / 4$, that intersects the disk intersects the vertical segment $p^{-} p^{+}$.

on $C$. We then use Proposition 2.1 to compute a shortest path between $p_{1}$ and $p_{n}$ in $G_{\varepsilon}$, thus solving the min-\# problem. Throughout this section, $d(\cdot, \cdot)$ denotes the distance under the $L_{1}$ metric.

We first give some definitions. We define the orientation of a line $\ell$, which we denote by $\Phi(\ell)$, to be the angle that $\ell$ makes with the positive $x$-axis in the range $[-\pi / 2, \pi / 2]$. We define the orientation of a line segment $p q$, which we denote by $\Phi(p q)$, to be the orientation of the line containing the line segment. Let $x(p)$ (resp. $y(p)$ ) denote the $x$-coordinate (resp. $y$-coordinate) of a point $p$. The following two lemmas state useful properties of the $L_{1}$ metric; see Figure 4.

Lemma 4.1. Let $\ell$ be a line such that $-\pi / 4 \leq \Phi(\ell) \leq \pi / 4$. For any point $p, d(p, \ell) \leq$ $\varepsilon$ under the $L_{1}$-metric if and only if $\ell$ intersects the vertical segment $p^{+} p^{-}$.

Lemma 4.2. Let $q r$ be a line segment such that $-\pi / 4 \leq \Phi(q r) \leq \pi / 4$, and assume $q$ lies to the left of $r$. For any point $p, d(p, q r) \leq \varepsilon$ if and only if exactly one of the following three conditions hold:

(i) $x(p)<x(q)$ and $d(p, q) \leq \varepsilon$.

(ii) $x(p)>x(r)$ and $d(p, r) \leq \varepsilon$.

(iii) $x(q) \leq x(p) \leq x(r)$ and $q r$ intersects the vertical segment $p^{+} p^{-}$.

As in Section 3, we use a divide-and-conquer approach to compute a clique cover of $G_{\varepsilon}(C)$. Let $C_{1}$ be the chain $\left\langle p_{1}, \ldots, p_{\lfloor n / 2\rfloor}\right\rangle$, and let $C_{2}$ be the chain $\left\langle p_{\lfloor n / 2\rfloor+1}, \ldots, p_{n}\right\rangle$. We recursively compute the clique covers $\mathcal{G}_{1}$ and $\mathcal{G}_{2}$ of $G_{\varepsilon}\left(C_{1}\right)$ and $G_{\varepsilon}\left(C_{2}\right)$, respectively. The merge step computes a clique cover $\mathcal{G}_{12}$ of the set

$$
E_{12}=\left\{\left(p_{i}, p_{j}\right) \in G_{\varepsilon} \mid p_{i} \in C_{1}, p_{j} \in C_{2}\right\} .
$$

We will describe an algorithm for computing a clique cover of the set of edges

$$
H_{12}=\left\{\left(p_{i}, p_{j}\right) \in E_{12} \mid x\left(p_{i}\right) \leq x\left(p_{j}\right),-\pi / 4 \leq \Phi\left(p_{i} p_{j}\right) \leq \pi / 4\right\} .
$$

By reversing the direction of the $(+x)$-axis and/or switching the role of the $x$ - and $y$ axis, we can compute a clique cover of the remaining edges of $E_{12}$. Our approach will 


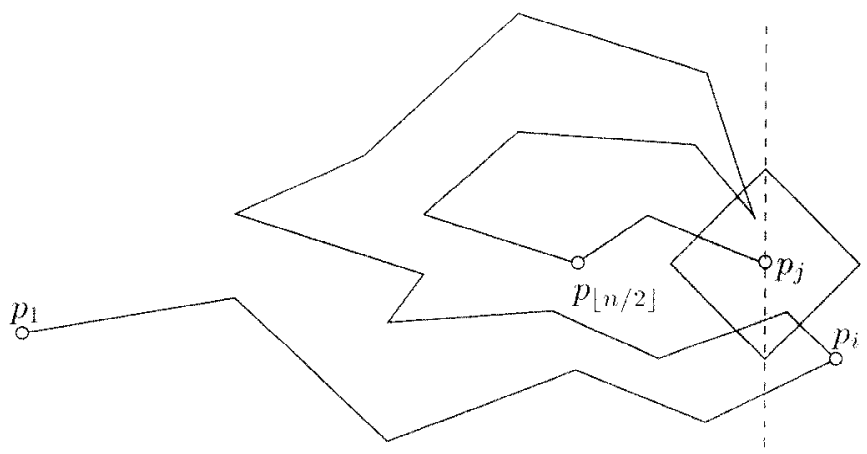

Fig. 5. $p_{i+1}, \ldots, p_{\lfloor n / 2\rfloor}$ are relevant for $p_{j} ; p_{1}, \ldots, p_{\lfloor n / 2\rfloor}$ are not relevant for $p_{j}$.

be to do some "filtering" so that we can compute a clique cover of $H_{12}$ by essentially the technique of Section 3 . Let $\bar{H}_{12}$ denote the following collection of pairs:

$$
\bar{H}_{12}=\left\{\left(p_{i}, p_{j}\right) \mid p_{i} \in C_{1}, p_{j} \in C_{2}, x\left(p_{i}\right) \leq x\left(p_{j}\right),-\pi / 4 \leq \Phi\left(p_{i} p_{j}\right) \leq \pi / 4\right\} .
$$

By definition, $H_{12} \subseteq \bar{H}_{12}$. We first compute a clique cover of $\bar{H}_{12}$, and then use that to compute a clique cover of $H_{12}$.

Definition 4.3. Let $p_{i} \in C_{1}$ and $p_{j} \in C_{2}$. We say that $p_{i}$ is relevant for $p_{j}$, if for all $p_{k}, i \leq k \leq\lfloor n / 2\rfloor$, such that $x\left(p_{k}\right)>x\left(p_{j}\right), d\left(p_{k}, p_{j}\right) \leq \varepsilon$. Similarly, we say that $p_{j} i s$ relevant for $p_{i}$ if for all $p_{k},\lfloor n / 2\rfloor+1 \leq k \leq j$, such that $x\left(p_{k}\right)<x\left(p_{i}\right), d\left(p_{k}, p_{i}\right) \leq \varepsilon$ (Fig. 5).

Definition 4.4. For $p_{i} \in C_{1}$, we define $\operatorname{cone}\left(p_{i}\right)$ to be the following cone of rightward directed rays emanating from $p_{i}$ : a ray $\rho$ belongs to cone $\left(p_{i}\right)$ if $-\pi / 4 \leq \Phi(\rho) \leq \pi / 4$ and $d\left(p_{k}, \rho\right) \leq \varepsilon$, for $i \leq k \leq\lfloor n / 2\rfloor$.

Symmetrically, we define cone $\left(p_{j}\right)$, for $p_{j} \in C_{2}$, to be the following cone of leftward directed rays emanating from $p_{j}$ : a ray $\rho$ belongs to cone $\left(p_{j}\right)$ if $-\pi / 4 \leq \Phi(\rho) \leq \pi / 4$ and $d\left(p_{k}, \rho\right) \leq \varepsilon$, for $\lfloor n / 2\rfloor+1 \leq k \leq j$.

By Lemma 4.2, a rightward directed ray $\rho$ originating at $p_{i}$ belongs to cone $\left(p_{i}\right)$ if and only if $-\pi / 4 \leq \Phi(\rho) \leq \pi / 4$, and, for every $i \leq k \leq\lfloor n / 2\rfloor$,

(i) if $x\left(p_{k}\right)<x\left(p_{i}\right)$, then $d\left(p_{k}, p_{i}\right) \leq \varepsilon$, and

(ii) if $x\left(p_{k}\right) \geq x\left(p_{i}\right)$, then $\rho$ intersects the vertical segment $p_{k}^{-} p_{k}^{+}$.

Similarly, a leftward directed ray $\rho$ originating at $p_{j}$ belongs to cone $\left(p_{j}\right)$ if and only if $-\pi / 4 \leq \Phi(\rho) \leq \pi / 4$, and, for every $\lfloor n / 2\rfloor+1 \leq k \leq j$,

(i) if $x\left(p_{k}\right)>x\left(p_{j}\right)$, then $d\left(p_{k}, p_{j}\right) \leq \varepsilon$, and

(ii) if $x\left(p_{k}\right) \leq x\left(p_{j}\right)$, then $\rho$ intersects the vertical segment $p_{k}^{-} p_{k}^{+}$.

The following lemma lays the foundation for our algorithm. 
Lemma 4.5. For any $\left(p_{i}, p_{j}\right) \in \bar{H}_{12},\left(p_{i}, p_{j}\right) \in H_{12}$ if and only if

(1) $p_{i}$ is relevant for $p_{j}$ and $p_{j}$ is relevant for $p_{i}$, and

(2) $p_{j} \in \operatorname{cone}\left(p_{i}\right)$ and $p_{i} \in \operatorname{cone}\left(p_{j}\right)$.

Proof. Let $\left(p_{i}, p_{j}\right) \in \bar{H}_{12}$, and assume that conditions (1) and (2) hold. To show that $\left(p_{i}, p_{j}\right) \in H_{12}$, we will show that $\left(p_{i}, p_{j}\right) \in G_{\varepsilon}$ by proving that for any $k, i \leq k \leq j$, $d\left(p_{k}, p_{i} p_{j}\right) \leq \varepsilon$. We consider three cases:

1. $x\left(p_{k}\right)<x\left(p_{i}\right)$. If $p_{k} \in C_{1}$, it must be the case that $d\left(p_{k}, p_{i}\right) \leq \varepsilon$, for otherwise cone $\left(p_{i}\right)$ would be empty. If $p_{k} \in C_{2}$, it follows from the fact that $p_{j}$ is relevant for $p_{i}$ that $d\left(p_{k}, p_{i}\right) \leq \varepsilon$. In either case, $d\left(p_{k}, p_{i} p_{j}\right) \leq \varepsilon$.

2. $x\left(p_{k}\right)>x\left(p_{j}\right)$. This case is symmetric to the earlier one.

3. $x\left(p_{i}\right) \leq x\left(p_{k}\right) \leq x\left(p_{j}\right)$. Assume that $p_{k} \in C_{1}$; the case where $p_{k} \in C_{2}$ is symmetric. Since $p_{j} \in$ cone $\left(p_{i}\right)$, the ray $R\left(p_{i}, p_{j}\right)$ emanating from $p_{i}$ and passing through $p_{j}$ is in cone $\left(p_{i}\right)$. By the definition of cone $\left(p_{i}\right)$, it must be the case that $d\left(p_{k}, R\left(p_{i}, p_{j}\right)\right) \leq \varepsilon$. Since $x\left(p_{k}\right) \geq x\left(p_{i}\right)$, it follows from Lemma 4.2 that $R\left(p_{i}, p_{j}\right)$ intersects the vertical segment $p_{k}^{-} p_{k}^{+}$. Moreover, since $x\left(p_{k}\right) \leq x\left(p_{j}\right)$, we can conclude that the segment $p_{i} p_{j}$ intersects the vertical segment $p_{k}^{-} p_{k}^{+}$. Thus, $d\left(p_{k}, p_{i} p_{j}\right) \leq \varepsilon$.

Now we establish the other half of the lemma. Assume that $\left(p_{i}, p_{j}\right) \in H_{12}$. We argue that (1) $p_{j}$ is relevant for $p_{i}$, and (2) the ray $R\left(p_{i}, p_{j}\right)$ lies in cone $\left(p_{i}\right)$. By symmetrical arguments, we can also show that $p_{i}$ is relevant for $p_{j}$ and the ray $R\left(p_{j}, p_{i}\right)$ lies in $\operatorname{cone}\left(p_{j}\right)$.

Consider any $p_{k}$ for $\lfloor n / 2\rfloor+1 \leq k \leq j$ such that $x\left(p_{k}\right)<x\left(p_{i}\right)$. Since $\left(p_{i}, p_{j}\right) \in G_{\varepsilon}$, $d\left(p_{k}, p_{i} p_{j}\right) \leq \varepsilon$. Lemma 4.2 tells us that this can only happen if $d\left(p_{k}, p_{i}\right) \leq \varepsilon$. (Here we are using the fact that $\left(p_{i}, p_{j}\right) \in \bar{H}_{12}$, and so $-\pi / 4 \leq \Phi\left(p_{i} p_{j}\right) \leq \pi / 4$.) We conclude that $p_{j}$ is relevant for $p_{i}$.

Consider any $p_{k}$ such that $i \leq k \leq\lfloor n / 2\rfloor$. Since $\left(p_{i}, p_{j}\right) \in G_{\varepsilon}$, it follows that $d\left(p_{k}, p_{i} p_{j}\right) \leq \varepsilon$, and hence $d\left(p_{k}, R\left(p_{i}, p_{j}\right)\right) \leq \varepsilon$. On the other hand, $\left(p_{i}, p_{j}\right) \in \bar{H}_{12}$, which imples that $R\left(p_{i}, p_{j}\right)$ is rightward directed and $-\pi / 4 \leq \Phi\left(R\left(p_{i}, p_{j}\right)\right) \leq \pi / 4$. We conclude that $R\left(p_{i}, p_{j}\right) \in$ cone $\left(p_{i}\right)$. This completes the proof of the second half of the lemma.

In view of Lemma 4.5, we compute a clique cover for $H_{12}$ in four steps.

Step 1 . We compute a clique cover of the edges in $\bar{H}_{12}$.

Step 2. For each bipartite clique $\left(A_{1}, A_{2}\right)$ computed in Step 2, we compute a clique cover of the pairs $\left(p_{i}, p_{j}\right) \in A_{1} \times A_{2}$ such that $p_{i}$ is relevant for $p_{j}$ and $p_{j}$ is relevant for $p_{i}$.

Step 3. For each $p_{i} \in C_{1}$, we compute cone $\left(p_{i}\right)$; for each $p_{j} \in C_{2}$, we compute cone $\left(p_{j}\right)$.

Step 4. For each bipartite clique $\left(B_{1}, B_{2}\right)$ computed in Step 3, we compute a clique cover of the pairs $\left(p_{i}, p_{j}\right) \in B_{1} \times B_{2}$ such that $p_{j} \in \operatorname{cone}\left(p_{i}\right)$ and $p_{i} \in \operatorname{cone}\left(p_{j}\right)$. This is done by taking the segments dual to the cones and proceeding exactly as in Section 3. 
The algorithms for Steps 1, 2, and 3 are described in Lemmas 4.7, 4.8, and 4.9, respectively. As described in Section 3, the procedure in Step 4 for a single bipartite clique $\left(B_{1}, B_{2}\right)$ can be implemented in $O\left(\left(\left|B_{1}\right|+\left|B_{2}\right|\right)^{4 / 3+\delta}\right)$. We conclude that we can compute a clique cover of $H_{12}$ of size $O\left(n^{4 / 3+\delta}\right)$ in $O\left(n^{4 / 3+\delta}\right)$ time. Putting everything together, we get an algorithm that computes a clique cover of $G_{\varepsilon}$ whose size is $O\left(n^{4 / 3+\delta}\right)$. The running time of the algorithm is also $O\left(n^{4 / 3+\delta}\right)$. We then use Proposition 2.1 to compute a shortest path between $p_{1}$ and $p_{n}$ in $G_{\varepsilon}(C)$, obtaining the main result of this section.

Theorem 4.6. For any $\delta>0$, we can solve the min-\# problem under the $L_{1}$ metric for $a$ (possibly) nonmonotone polygonal chain in $O\left(n^{4 / 3+\delta}\right)$ time.

In the rest of this section we describe the procedures for Steps 1, 2, and 3 in detail.

Lemma 4.7. We can compute, in $O\left(n \log ^{2} n\right)$ time, a clique cover of $\bar{H}_{12}$ of size $O\left(n \log ^{2} n\right)$.

Proof. For a point $p$ in the plane, let

$$
\operatorname{Wed}(p)=\left\{q \in \mathbb{R}^{2}|| y(p)-y(q) \mid \leq x(q)-x(p)\right\},
$$

that is, $\operatorname{Wed}(p)$ is the wedge that is bounded by the two rightward directed rays emanating from $p$ with orientations $+\pi / 4$ and $-\pi / 4$. By definition of $\bar{H}_{12},\left(p_{i}, p_{j}\right) \in \bar{H}_{12}$ if and only if $p_{j} \in \operatorname{Wed}\left(p_{i}\right)$. We preprocess $C_{2}$ into a data structure to answer queries of the following form efficiently: Given a point $p$, report all points $q \in C_{2}$ that lie in $\operatorname{Wed}(p)$.

The data structure we construct is a range-tree [34] on $C_{2}$, which can report the points in $C_{2}$ that lie in a query wedge $\operatorname{Wed}(p)$ as a union of $O\left(\log ^{2} n\right)$ disjoint canonical subsets of $C_{2}$. The total size of all the canonical subsets in the range tree is $O(n \log n)$. With this data structure, we proceed as in Section 3 to construct a clique cover of $\bar{H}_{12}$. That is, we query the data structure with all points $p \in C_{1}$. For each canonical subset $B_{i}$ in the range tree, let $A_{i} \subseteq C_{1}$ be the set of query points whose output contains $B_{i}$. If $A_{i} \neq \varnothing$, we include $\left(A_{i}, B_{i}\right)$ as a bipartite clique of the clique cover. The size of the clique cover is $O\left(n \log ^{2} n\right)$, and the running time is also $O\left(n \log ^{2} n\right)$.

Lemma 4.8. Let $A \subseteq C_{1}$ and $B \subseteq C_{2}$, and let $|A|+|B|=m$. Let $\operatorname{Rel}(A, B) \subseteq A \times B$ denote the collection of ordered pairs $\left(p_{i}, p_{j}\right)$ such that $p_{i}$ is relevant for $p_{j}$ and $p_{j}$ is relevant for $p_{i}$. We can compute a clique cover of $\operatorname{Rel}(A, B)$ of size $O\left(m \log ^{2} m\right)$ in $O\left(m \log ^{6} m\right)$ time.

Proof. We proceed in two stages. In the first stage we compute a clique cover $\mathcal{G}^{\prime}$ for the ordered pairs $\left(p_{i}, p_{j}\right) \in A \times B$ such that $p_{i}$ is relevant for $p_{j}$. We assume that $A$ is given as a sequence ordered according to $C_{1}$; that is, $p_{i} \in A$ occurs before $p_{k} \in A$ if $i<k$. For $p_{j} \in B$, let $\alpha\left(p_{j}\right)$ be the last point in the sequence $A$ such that $x\left(\alpha\left(p_{j}\right)\right)>x\left(p_{j}\right)$ and $d\left(\alpha\left(p_{j}\right), p_{j}\right)>\varepsilon$. Observe that the points in the sequence $A$ after $\alpha\left(p_{j}\right)$ are the ones that are relevent for $p_{j}$. Thus the set of points in $A$ that are relevant for a given $p_{j} \in B$ forms a suffix of the sequence $A$. Exploiting this observation, we can compute, 
in a straightforward fashion, a collection of canonical subsets of $A$, whose total size is $O(m \log m)$, so that, for any $p_{j} \in B$, the set of points in $A$ that are relevant for $p_{j}$ can be returned as a disjoint union of $O(\log m)$ canonical sets. With this data structure, we proceed as in the proof of Lemma 4.7 to construct a clique cover $\mathcal{G}^{\prime}$ whose size is $O(m \log m)$. The time for computing the clique cover is governed by the time needed to compute $\alpha\left(p_{j}\right)$ for each $p_{j} \in B$; combining binary search with a data structure based on range trees [34], this can be done in a total of $O\left(m \log ^{5} m\right)$ time.

In the second stage we take each bipartite clique $\left(A^{\prime}, B^{\prime}\right)$ of $\mathcal{G}_{1}$, and compute a clique cover of the set of ordered pairs $\left(p_{i}, p_{j}\right) \in A^{\prime} \times B^{\prime}$ such that $p_{j}$ is relevant for $p_{i}$. We do this in a manner completely similar and symmetric to the first stage. If $\left|A^{\prime}\right|+\left|B^{\prime}\right|=m^{\prime}$, the second stage applied to $\left(A^{\prime}, B^{\prime}\right)$ produces a clique cover of size $O\left(m^{\prime} \log m^{\prime}\right)$.

We return the union of all the clique covers computed after the second stage as the clique cover of $\operatorname{Rel}(A, B)$. The size of the clique cover is $O\left(m \log ^{2} m\right)$, and the overall running time is $O\left(m \log ^{6} m\right)$.

Lemma 4.9. We can compute cone $\left(p_{i}\right)$, for $1 \leq i \leq n$, in $O\left(n \log ^{3} n\right)$ time.

Proof. We only describe the computation of cone $\left(p_{i}\right)$ for $p_{i} \in C_{1}$. Let left $\left(p_{i}\right)$ (resp. $\left.\operatorname{right}\left(p_{i}\right)\right)$ be the set of points $p_{k}, i \leq k \leq\lfloor n / 2\rfloor$, such that $x\left(p_{k}\right)<x\left(p_{i}\right)\left(\right.$ resp. $x\left(p_{k}\right) \geq$ $x\left(p_{i}\right)$ ). To compute cone $\left(p_{i}\right)$, we first test if $d\left(p_{i}, p\right) \leq \varepsilon$, for every $p \in \operatorname{left}\left(p_{i}\right)$. If, for any $p \in \operatorname{left}\left(p_{i}\right), d\left(p_{i}, p\right)>\varepsilon$, we stop and declare cone $\left(p_{i}\right)$ to be empty. Otherwise, we compute the cone of rightward directed rays emanating from $p_{i}$ consisting of all rays $\rho$ such that $\rho$ intersects the vertical segment $p^{-} p^{+}$, for every $p \in \operatorname{right}\left(p_{i}\right)$. We clip this cone so that all rays of this cone have orientation between $-\pi / 4$ and $\pi / 4$, and return the clipped cone as cone $\left(p_{i}\right)$.

In order to do all this efficiently, we preprocess $C_{1}$ into a data structure that returns $\operatorname{left}\left(p_{i}\right)$ (or $\left.\operatorname{right}\left(p_{i}\right)\right)$, for any $p_{i} \in C_{1}$ as a union of $O\left(\log ^{2} n\right)$ canonical subsets. This data structure is simply the two-dimensional range tree in which the points are ordered in one dimension according to their $x$-coordinates, and in the other dimension according to their occurrence in the chain $C_{1}$ (that is, $p_{1}, \ldots, p_{\lfloor n / 2\rfloor}$ ). With each canonical subset $S$ of the data structure, we store the region $\operatorname{reg}(S)=\{q \mid d(q, p) \leq \varepsilon$, for all $p \in S\}$. It is easy to see that $\operatorname{reg}(S)$ is a rectangle. We also store the upper boundary $U(S)$ of the convex hull of the points $\left\{p^{-} \mid p \in S\right\}$ and the lower boundary $L(S)$ of the points $\left\{p^{+} \mid p \in S\right\}$. The data structure can be built in $O\left(n \log ^{3} n\right)$ time.

With these data structures set up, we can compute cone $\left(p_{i}\right)$, for any $p_{i} \in C_{1}$, as follows. We query the data structure and find $O\left(\log ^{2} n\right)$ canonical subsets $\left\{S_{1}, \ldots, S_{l}\right\}$ whose union is $\operatorname{left}\left(p_{i}\right)$. For each $S_{k}$, we check whether $p_{i} \in \operatorname{reg}\left(S_{k}\right)$; if, for any $S_{k}$, $p_{i} \notin S_{k}$, we stop and declare cone $\left(p_{i}\right)$ to be empty. Otherwise, we query the data structure to find $O\left(\log ^{2} n\right)$ canonical $\left\{S_{1}, \ldots, S_{l}\right\}$ subsets whose union is $\operatorname{right}\left(p_{i}\right)$. For each $S_{k}$, we compute the cone of rightward directed rays emanating from $p_{i}$ that is bounded on the top by the ray from $p_{i}$ tangent to $L\left(S_{k}\right)$, and on the bottom by the ray from $p_{i}$ tangent to $U\left(S_{k}\right)$. We compute the intersection of the cones computed for each $S_{k}$. Finally, we clip this cone to one whose rays have orientation between $-\pi / 4$ and $\pi / 4$.

Using this procedure, we can compute $\operatorname{cone}\left(p_{i}\right)$ for any $p_{i} \in C_{1}$ in $O\left(\log ^{3} n\right)$ time. Hence, the overall algorithm for computing all the cones runs in $O\left(n \log ^{3} n\right)$ time. 


\section{The Min- $\varepsilon$ Algorithm under the $L_{1}$ Metric}

In the min- $\varepsilon$ problem we are given a polygonal chain $C=\left\langle p_{1} \cdots p_{n}\right\rangle$ and an integer $k \leq n$, and we want to find an approximation of $C$ that minimizes the error over all approximations that use at most $k$ vertices. In this section we present a randomized algorithm to solve the min- $\varepsilon$ problem for a polygonal chain $C$ under the $L_{1}$ metric. As mentioned in Section 2, the min- $\varepsilon$ problem reduces to finding the smallest value of $\varepsilon$, for which there is a path in $G_{\varepsilon}$ between $p_{1}$ and $p_{n}$ consisting of at most $k$ vertices; we let $\varepsilon^{*}$ denote this smallest value. Recall that $\varepsilon^{*}=\Delta\left(p_{i} p_{j}\right)$, the error of some segment $p_{i}, p_{j}$. Given an $\varepsilon$, we can use the min-\# algorithm as a decision procedure to determine whether $\varepsilon^{*} \leq \varepsilon$ or $\varepsilon^{*}>\varepsilon$. Hence, we can use the decision procedure to binary search the errors corresponding to each of the $\Theta\left(n^{2}\right)$ segments $p_{i} p_{j}$. However, we cannot explicitly enumerate these errors if we are aiming for a subquadratic algorithm. Instead, we use a variant of the random halving technique [30] to do the search. Our algorithm can be made deterministic using the expander based approach by Katz and Sharir [27]. We first describe some primitives that our algorithm uses.

Lemma 5.1. For any given $\varepsilon \geq 0$, we can preprocess the polygonal chain $C$ into a data structure in $O\left(n^{4 / 3+\delta}\right)$ time, for any $\delta>0$, so that we can select with uniform probability a random segment $p_{i} p_{j}$ whose error is at most $\varepsilon$. The selection procedure takes $O(\log n)$ time.

Proof. For simplicity, we assume that we have access to a random number generator that can generate a random number in the range $(0,1]$ with uniform probability. We first compute a clique cover $\mathcal{G}=\left\{G_{1}=\left(V_{1}, E_{1}\right), \ldots, G_{l}=\left(V_{l}, E_{l}\right)\right\}$ of the graph $G_{\varepsilon}$ in $O\left(n^{4 / 3+\delta}\right)$ time. Let $A_{i}$ and $B_{i}$ denote the vertex classes of $V_{i}$. Let

$$
w=\sum_{1 \leq i \leq l}\left|A_{i}\right| *\left|B_{i}\right|
$$

denote the total number of segments with error at most $\varepsilon$. We first describe how we can select a bipartite clique $G_{i}$ with probability $w_{i}=\left|A_{i}\right|\left|B_{i}\right| / w$. We divide the interval $(0,1]$ into intervals $I_{1}, \ldots, I_{l}$, where

$$
I_{j}=\left(\sum_{1 \leq k \leq j-1} w_{k}, \sum_{1 \leq k \leq j} w_{k}\right] .
$$

To generate a random bipartite clique, we first generate a random number $r \in(0,1]$. We do a binary search on the intervals $I_{1}, \ldots, I_{l}$ to locate the interval $I_{j}$ containing $r$, and return $G_{j}$.

For each $A_{i}$ (resp. $B_{i}$ ), we build a similar structure that will allow us to generate an element $a \in A_{i}$ (resp. $b \in B_{i}$ ) with probability $1 /\left|A_{i}\right|$ (resp. $1 /\left|B_{i}\right|$ ). To pick a random segment $p_{i} p_{j}$ with error at most $\varepsilon$, we first pick a random bipartite clique as above; if $G_{k}$ is picked, we pick random elements $a \in A_{k}$ and $b \in B_{k}$, and return $(a, b)$.

The preprocessing time of this scheme is $O\left(n^{4 / 3+\delta}\right)$, and the time for generating a random segment is $O(\log n)$. If $\Delta\left(p_{i} p_{j}\right) \leq \varepsilon$, and $\left(p_{i}, p_{j}\right) \in A_{k} \times B_{k}$, the probability of picking $\left(p_{i}, p_{j}\right)$ is

$$
w_{k} * 1 /\left|A_{k}\right| * 1 /\left|B_{k}\right|=1 / w
$$


Using clique covers, the following lemma is easily established.

Lemma 5.2. For any given $\varepsilon \geq 0$, we can count, in $O\left(n^{4 / 3+\delta}\right)$ time, for any $\delta>0$, the number of segments $p_{i} p_{j}$ whose error is at most $\varepsilon$. As a corollary, we can count in $O\left(n^{4 / 3+\delta}\right)$ time the number of segments $p_{i} p_{j}$ whose error lies in a given range $\left(\varepsilon_{1}, \varepsilon_{2}\right]$.

Proof. We compute a clique cover $\mathcal{G}=\left\{G_{1}=\left(V_{1}, E_{1}\right), \ldots, G_{l}=\left(V_{l}, E_{l}\right)\right\}$ of the graph $G_{\varepsilon}$. Let $A_{i}$ and $B_{i}$ denote the vertex classes of $V_{i}$. Then the total number of segments with error at most $\varepsilon$ is

$$
\sum_{1 \leq i \leq l}\left|A_{i}\right| *\left|B_{i}\right| .
$$

The number of segments whose error lies in the range $\left(\varepsilon_{1}, \varepsilon_{2}\right]$ is the number of segments whose error is at most $\varepsilon_{2}$ minus the number of segments whose error is at most $\varepsilon_{1}$.

We will also need the following lemma.

Lemma 5.3. We can preprocess the polygonal chain $C$ in $O\left(n \log ^{2} n\right)$ time so that given a query segment $p_{i} p_{j}$, its error can be computed in $O\left(\log ^{3} n\right)$ time.

Proof. We only describe the data structure for query segments $p_{i} p_{j}$ that belong to

$$
\bar{H}=\left\{p_{i} p_{j} \mid x\left(p_{i}\right) \leq x\left(p_{j}\right),-\pi / 4 \leq \Phi\left(p_{i} p_{j}\right) \leq \pi / 4\right\} .
$$

Let

$$
\begin{aligned}
\operatorname{left}\left(p_{i}, p_{j}\right) & =\left\{p_{k} \mid i \leq k \leq j, x\left(p_{k}\right)<x\left(p_{i}\right)\right\}, \\
\operatorname{right}\left(p_{i}, p_{j}\right) & =\left\{p_{k} \mid i \leq k \leq j, x\left(p_{k}\right)>x\left(p_{j}\right)\right\}, \quad \text { and } \\
\operatorname{between}\left(p_{i}, p_{j}\right) & =\left\{p_{k} \mid i \leq k \leq j, x\left(p_{i}\right) \leq x\left(p_{k}\right) \leq x\left(p_{j}\right)\right\} .
\end{aligned}
$$

We preprocess the points $\left\{p_{1}, \ldots, p_{n}\right\}$ in $O(n \log n)$ time into a data structure so that given any $\left(p_{i}, p_{j}\right), \operatorname{left}\left(p_{i}, p_{j}\right), \operatorname{right}\left(p_{i}, p_{j}\right)$, and between $\left(p_{i}, p_{j}\right)$ can be returned as a union of $O\left(\log ^{2} n\right)$ canonical subsets. This data structure is simply the two-dimensional range tree in which the points are ordered in one dimension according to their $x$ coordinates, and in the other dimension according to their occurrence in the chain $C$ (that is, $p_{1}, \ldots, p_{n}$ ). For each canonical set $S \subseteq C$ of the data structure, we store $\operatorname{conv}(S)$, the convex hull of $S$. We also store $\operatorname{extr}(S)$, the four " $L_{1}$-extremal" points of $S$; for any point $p$ in the plane, the point in $S$ that maximizes the $L_{1}$ distance to $p$ is one of these four points.

This completes the description of our data structure for query segments in $\bar{H}$. Recall that $\Delta\left(p_{i} p_{j}\right)=\max _{i \leq k \leq j} d\left(p_{k}, p_{i} p_{j}\right)$. For $p_{i} p_{j} \in \bar{H}, d\left(p_{k}, p_{i} p_{j}\right)$ equals $d\left(p_{k}, p_{i}\right)$ (resp. $\left.d\left(p_{k}, p_{j}\right)\right)$ for $p_{k} \in \operatorname{left}\left(p_{i}, p_{j}\right)$ (resp. for $\left.p_{k} \in \operatorname{right}\left(p_{i}, p_{j}\right)\right)$, and equals the vertical distance between $p_{k}$ and the line $\ell$ through $p_{i}$ and $p_{j}$ for $p_{k} \in \operatorname{between}\left(p_{i}, p_{j}\right)$ because $-\pi / 4 \leq \Phi\left(p_{i} p_{j}\right) \leq \pi / 4$. It follows that $\Delta\left(p_{i} p_{j}\right)$ is determined by either the point $q_{1}$ in left $\left(p_{i}, p_{j}\right)$ that maximizes the distance to $p_{i}$, or the point $q_{2}$ in $\operatorname{right}\left(p_{i}, p_{j}\right)$ 
that maximizes the distance to $p_{j}$, or the point in $q_{3}$ between $\left(p_{i}, p_{j}\right)$ that maximizes the vertical distance to the line $\ell$.

Our query procedure for computing $\Delta\left(p_{i} p_{j}\right)$ computes $q_{1}, q_{2}$, and $q_{3}$ as follows. We first query the above data structure to find the $O\left(\log ^{2} n\right)$ canonical sets whose union is left $\left(p_{i}, p_{j}\right)$. For each canonical set $S$, we find the point in $S$ maximizing the distance to $p_{i}$ by looking at the four $L_{1}$-extremal points of $S$. Thus, we can determine $q_{1}$ in $O\left(\log ^{2} n\right)$ time. By a symmetric scheme, we can also determine $q_{2}$ in $O\left(\log ^{2} n\right)$ time. To determine $q_{3}$, we query the above data structure to find $O\left(\log ^{2} n\right)$ canonical sets whose union is between $\left(p_{i}, p_{j}\right)$. For each canonical set $S$, we find the point in $S$ maximizing the vertical distance to $\ell$ by doing a binary search over the convex hull conv $(S)$ of $S$. The binary search finds the two lines $\ell^{\prime}$ and $\ell^{\prime \prime}$ that are parallel to $\ell$ and tangent to $\operatorname{conv}(S)$. If $p^{\prime} \in S$ (resp. $p^{\prime \prime} \in S$ ) is the point through which $\ell^{\prime}$ (resp. $\ell^{\prime \prime}$ ) passes, then either $p^{\prime}$ or $p^{\prime \prime}$ maximizes the vertical distance from $S$ to $\ell$. Thus, we can determine $q_{3}$ in $O\left(\log ^{3} n\right)$ time. The time for the overall query procedure is also $O\left(\log ^{3} n\right)$.

The Algorithm. The min- $\varepsilon$ algorithm maintains a working interval $I=\left(\varepsilon_{1}, \varepsilon_{2}\right]$ containing $\varepsilon^{*}$. We refer to the segments $\left(p_{i}, p_{j}\right)$ whose errors lie in the working interval as the candidate segments, and the corresponding errors as the candidate values. Our algorithm operates in two phases. In the first phase we repeatedly shrink the working interval containing $\varepsilon^{*}$ until it contains at most $t=\left\lfloor n^{2 / 3}\right\rfloor$ candidate values. In the second phase we expicitly enumerate all the segments whose errors lie in the working interval, and binary search the errors to find $\varepsilon^{*}$. We can afford to do the explicit enumeration because the working interval does not contain too many candidate values. We now describe the phases in detail.

The First Phase. The first phase works in stages. Suppose that at the beginning of the $i$ th stage, we have a working interval $I^{i-1}=\left(\varepsilon_{1}, \varepsilon_{2}\right]$ that contains $\varepsilon^{*}$. (Before beginning the first stage, we check whether $\varepsilon^{*}=0$ using the decision procedure. If $\varepsilon^{*}>0$, we set $I^{0}=(0, \infty]$.

1. Let $N^{i-1}$ denote the set of the candidate values contained in $I^{i-1}$. We check whether $\left|N^{i-1}\right| \leq t$ using the algorithm of Lemma 5.2. If $\left|N^{i-1}\right| \leq t$, the first phase ends and we proceed to the second phase. Otherwise, we shrink the working interval, as described below, to an interval $I^{i}$ such that $\left|N^{i}\right| \leq\left|N^{i-1}\right| / 3$.

2. We run the preprocessing algorithm of Lemma 5.1 that will allow us to generate a random segment $p_{i} p_{j}$ whose error lies in the range $\left(0, \varepsilon_{2}\right]$.

3. We generate, in $O(\log n)$ time, a random segment $p_{i} p_{j}$ whose error lies in the range $\left(0, \varepsilon_{2}\right]$. We compute its error $\varepsilon^{\prime}=\Delta\left(p_{i} p_{j}\right)$ in $O\left(\log ^{3} n\right)$ time using Lemma 5.3. If $\varepsilon^{\prime}$ does not lie in the working interval $I_{i-1}$ (i.e., $\varepsilon^{\prime}<\varepsilon_{1}$ ), we repeat this step.

4. Otherwise $\left(\varepsilon^{\prime} \in I_{i-1}\right)$, we check if $\varepsilon^{\prime}$ lies in the middle third of the values in $N^{i-1}$. We do this in $O\left(n^{4 / 3+\delta}\right)$ time by using the algorithm of Lemma 5.2 to count the number of candidate values in the intervals $\left(\varepsilon_{1}, \varepsilon^{\prime}\right]$ and $\left(\varepsilon^{\prime}, \varepsilon_{2}\right]$. If $\varepsilon^{\prime}$ does not lie in the middle third, we go back to Step 3.

5. We use the min-\# algorithm to decide if $\varepsilon^{*}>\varepsilon^{\prime}$, or $\varepsilon^{*} \leq \varepsilon^{\prime}$. If $\varepsilon^{*}>\varepsilon^{\prime}$, we let the working interval for the next stage be $I^{i}=\left(\varepsilon^{\prime}, \varepsilon_{2}\right]$; otherwise we let $I^{i}=\left(\varepsilon_{1}, \varepsilon^{\prime}\right]$. 
Clearly, a random candidate value in the interval $I_{i-1}$ lies in the middle third of the values in $N_{i-1}$ with probability at least $1 / 3$. Since $N_{i-1}$ contains at least $t$ values, a random segment $p_{i} p_{j}$ generated in Step 3 lies in $I_{i-1}$ with probability at least $t / n^{2} \approx 1 / n^{4 / 3}$. Using these observations, it is easy to see that with high probability (probability at least $1-1 / n^{c}$, for some constant $\left.c\right)$, there are $O\left(n^{4 / 3} \log n\right)$ iterations of Step 3 and $O(\log n)$ iterations of Step 4 in the $i$ th stage. We can conclude that the time taken to execute the $i$ th stage is $O\left(n^{4 / 3+\delta}\right)$, with high probability. Since, during each stage, the number of candidate values in the working interval decreases by a constant fraction, there are only a logarithmic number of stages. Therefore, the overall running time of the first phase is $O\left(n^{4 / 3+\delta}\right)$, with high probability.

The Second Phase. Assume that we enter the second phase with the working interval $I=\left(\varepsilon_{1}, \varepsilon_{2}\right]$. We first compute a superset of such segments and then discard those whose values do not lie in $I$. We compute the candidate segments and values that lie in $I$ as follows. We compute the clique covers of $G_{\varepsilon_{1}}$ and $G_{\varepsilon_{2}}$, and use them to find the in-degree and out-degree of each vertex in $G_{\varepsilon_{1}}$ and $G_{\varepsilon_{2}}$; this takes $O\left(n^{4 / 3+\delta}\right)$ time. We then find the set $S_{1}$ (resp. $S_{2}$ ) of all vertices $p_{i}$ (resp. $p_{j}$ ) of $C$ for which there is some $p_{j}$ (resp. $p_{i}$ ), with $i<j$, such that the error of segment $p_{i} p_{j}$ lies in $I$. A vertex $p_{i}$ belongs to $S_{1}$ (resp. $S_{2}$ ) if its out-degree (resp. in-degree) in $G_{\varepsilon_{1}}$ is strictly smaller than its out-degree (resp. in-degree) in $G_{\varepsilon_{2}}$. Hence, we can find $S_{1}$ (resp. $S_{2}$ ) by comparing the out-degrees (resp. in-degrees) of each vertex in $G_{\varepsilon_{1}}$ and $G_{\varepsilon_{2}}$. Clearly, $S_{1}$ and $S_{2}$ contain at most $t$ vertices. Using Lemma 5.3, we compute the error of each $\left(p_{i}, p_{j}\right) \in S_{1} \times S_{2}$, and discard the segments whose errors do not lie in $I$. We are left with the segments whose errors lie in $I$. Finally, we perform a binary search over the $O\left(n^{2 / 3}\right)$ candidate values in $I$, using the min-\# algorithm as a decision procedure, to compute $\varepsilon^{*}$. The overall running time of the second phase is also bounded by $O\left(n^{4 / 3+\delta}\right)$. Hence, we can conclude:

Theorem 5.4. For any $\delta>0$, we can solve the min- $\varepsilon$ problem under the $L_{1}$ metric using a randomized algorithm with expected running time $O\left(n^{4 / 3+\delta}\right)$.

\section{Conclusions}

We have presented efficient algorithms that exploit the structure of the graph of short-cuts $G_{\varepsilon}$. This brings us to an extremely interesting question: What happens when other error criteria are used? (See [25].) An interesting case is when the Euclidean distance is used to define the error. We have not been able to extend the techniques used in this paper to compute a compact representation of the graph $G_{\varepsilon}$ in this case.

Another interesting problem is to find near-linear-time algorithms for the problems solved in this paper.

\section{References}

1. P. K. Agarwal, N. Alon, B. Aronov, and S. Suri. Can visibility graphs be represented compactly? Discrete Comput. Geom., 12:347-365, 1994.

2. P. K. Agarwal and M. Sharir. Applications of a new space-partitioning technique. Discrete Comput. Geom., 9:11-38, 1993. 
3. A. Aggarwal, H. Booth, J. O'Rourke, S. Suri, and C. K. Yap. Finding minimal convex nested polygons. Inform. Comput., 83(1):98-110, Oct. 1989.

4. N. Alon and J. Spencer. The Probabilistic Method. Wiley, New York, 1993.

5. T. Asano and N. Katoh. Number theory helps line detection in digital images. In Proc. 4th Annual International Symposium on Algorithms and Computing, volume 762 of Lecture Notes in Computer Science, pages 313-322. Springer-Verlag, Berlin, 1993.

6. R. E. Bellman and R. S. Roth. Methods in Approximation: Techniques for Mathematical Modelling. Reidel, Boston, MA, 1986.

7. B. Buttenfield. Treatment of the cartographic line. Cartographica, 22:1-26, 1985.

8. W. S. Chan and F. Chin. Approximation of polygonal curves with minimum number of line segments. In Proc. 3rd Annual International Symposium on Algorithms and Computing, volume 650 of Lecture Notes in Computer Science, pages 378-387. Springer-Verlag, Berlin, 1992.

9. S. D. Conte and C. de Boor. Elementary Numerical Analysis: An Algorithmic Approach, 3rd edn. McGrawHill, New York, 1980.

10. T. H. Cormen, C. E. Leiserson, and R. L. Rivest. Introduction to Algorithms. MIT Press, Cambridge, MA, 1990.

11. R. G. Cromley. A vertex substitution approach to numerical line simplification. In Proc. 3 rd International Symposium Spatial Data Handling, pages 57-64, 1988.

12. P. J. Davis. Interpolation and Approximation. Blaisdell, New York, 1963.

13. M. de Berg, M. van Kreveld, and S. Schirra. A new approach to subdivision simplification. In Proc. 12th International Symposium on Computer-Assisted Cartography, pages 79-88, 1995.

14. P. Dierckx. Curve and Surface Fitting with Splines. Clarendon Press, New York, 1993.

15. D. H. Douglas and T. K. Peucker. Algorithms for the reduction of the number of points required to represent a digitized line or its caricature. Canad. Cartog., 10(2):112-122, Dec. 1973.

16. D. Eu and G. T. Toussaint. On approximating polygonal curves in two and three dimensions. CVGIP: Graph. Models Image Process., 56(3):231-246, May 1994.

17. T. Feder and R. Motwani. Clique partitions, graph compression, and speeding up algoithms. In Proc. 27th Annual ACM Symposium on Theory of Computing, pages 123-133, 1991.

18. M. T. Goodrich. Efficient piecewise-linear function approximation using the uniform metric. Discrete Comput. Geom., 14:445-462, 1995.

19. L. J. Guibas, J. E. Hershberger, J. S. B. Mitchell, and J. S. Snoeyink. Approximating polygons and subdivisions with minimum link paths. Internat. J. Comput. Geom. Appl., 3(4):383-415, Dec. 1993.

20. S. L. Hakimi and E. F. Schmeichel. Fitting polygonal functions to a set of points in the plane. CVGIP: Graph. Models Image Process., 53(2):132-136, 1991.

21. J. Hershberger and J. Snoeyink. Speeding up the Douglas-Peucker line simplification algorithm. In Proc. 5th International Symposium on Spatial Data Handling, pages 134-143, 1992.

22. J. Hershberger and J. Snoeyink. Computing minimum length paths of a given homotopy class. Comput. Geom. Theory Appl., 4:63-98, 1994.

23. J. Hershberger and J. Snoeyink. Cartographic line simplification and polygon CSG formulae in $O(n \log * n)$ time. In Proc. 5th International Workshop on Algorithms and Data Structures, volume 1272 of Lecture Notes in Computer Science, pages 93-103. Springer-Verlag, Berlin, 1997.

24. J. D. Hobby. Polygonal approximations that minimize the number of inflections. In Proc. 4th ACM-SIAM Symposium on Discrete Algorithms, pages 93-102, 1993.

25. H. Imai and M. Iri. Polygonal approximations of a curve-formulations and algorithms. In G. T. Toussaint, editor, Computational Morphology, pages 71-86. North-Holland, Amsterdam, 1988.

26. S. Kahan and J. Snoeyink. On the bit complexity of minimum link paths: superquadratic algorithms for problems solvable in linear time. In Proc. 12th Annual ACM Symposium on Computational Geometry, pages 151-158, 1996.

27. M. J. Katz and M. Sharir. An expander-based approach to geometric optimization. SIAM J. Comput., 26:1384-1408, 1997.

28. Y. Kurozumi and W. A. Davis. Polygonal approximation by the minimax method. Comput. Graph. Image Process., 19:248-264, 1982.

29. Z. Li and S. Openshaw. Algorithms for automated line generalization based on a natural principle of objective generalization. Internat. J. Geogr. Inform. Systems, 6:373-389, 1992.

30. J. Matoušek. Randomized optimal algorithm for slope selection. Inform. Process. Lett., 39:183-187, 1991. 
31. R. B. McMaster. Automated line generation. Cartographica, 24(2):74-111, 1987.

32. A. Melkman. On-line construction of the convex hull of a simple polyline. Inform. Process. Lett., 25:11-12, 1987.

33. A. Melkman and J. O'Rourke. On polygonal chain approximation. In G. T. Toussaint, editor, Computational Morphology, pages 87-95. North-Holland, Amsterdam, 1988.

34. F. P. Preparata and M. I. Shamos. Computational Geometry: An Introduction. Springer-Verlag, New York, 1985.

35. S. Suri. A linear time algorithm for minimum link paths inside a simple polygon. Comput. Vision Graph. Image Process., 35:99-110, 1986.

36. G. T. Toussaint. On the complexity of approximating polygonal curves in the plane. In Proc. IASTED, International Symposium on Robotics and Automation, 1985.

Received September 17, 1998, and in revised form July 8, 1999. 